\title{
Dissemination and Innovation of Chinese Excellent Traditional Culture under the Background of New Media
}

\author{
Shang Xiao-mei \\ Wuhan Business University \\ Wuhan, Hubei Provience, 430056
}

\author{
Li Yao-nan* \\ Wuhan Business University \\ Wuhan, Hubei Provience, 430056
}

\begin{abstract}
In the era of new media, the overall situation of the dissemination of excellent traditional Chinese culture is poor. It is mainly manifested in the lack of dissemination, lack of influence, content polarization, and single form of communication. The dissemination of new media of the fine traditional Chinese culture should give full play to the advantages of the new media and adhere to the core values of socialism. Under the premise of guidance, on the basis of respecting the basic characteristics of traditional culture, the modern conversion of expressions will be the breakthrough point. Through the theme planning, we will search for the point of convergence between the traditional spirit and the spirit of the times, and make full use of its interactive function to spread the audience and the disseminator. Converted to the focus of efforts to gradually reverse the situation of the dissemination of new media in the fine traditional Chinese culture.
\end{abstract}

Keywords-New media; Excellent traditional Chinese culture; Dissemination; Innovation

\section{INTRODUCTION}

With the rapid development of the Internet and digital technologies, new media represented by WeChat, Weibo, APP, etc. have developed rapidly. They have profoundly influenced and changed people's communication methods, information acquisition channels, learning reading habits, and consumer shopping. , entertainment, etc. Against this backdrop, globalization has become more and more closely linked, the speed of information transmission and the scale of communication have accelerated, and the collisions between different cultures and value systems have become increasingly frequent. The tremendous changes in the media environment have presented tremendous challenges to the succession, development, and innovation of the excellent Chinese traditional culture. How to expand the influence of excellent Chinese traditional culture and display its permanent charm and era style is the core issue of the dissemination of excellent Chinese traditional culture in the era of new media.

\section{ANALYSIS OF THE STATUS QUO AND CAUSES OF THE DISSEMINATION OF EXCELLENT TRADITIONAL CHINESE CULTURE IN THE ERA OF NEW MEDIA}

The overall status quo of the dissemination of excellent traditional culture in China is poor, mainly reflected in the following points:

The first is that the new media does not have enough communication power and has insufficient influence. At present, the mainstay of traditional culture still focuses on traditional media such as newspapers and television. The new media have a serious shortage in disseminating traditional culture. Taking the WeChat public number as an example, the number of public numbers with traditional culture as the main content is very small, and there are very few in terms of the number of tweets, the number of fans and the amount of reading.

The second is that there are two extremes in the dissemination of content, which are academic and professional, and there is a small "gap" between the cultural needs of the general public and the inability to meet the needs of entertainment and leisure; or they are too entertaining. Cheating, for the sake of blog flow and eyeballs, makes traditional culture "taste". For example, some of the public names under the name of "Chinese Culture" and "Traditional Culture" have emphasized the sharing of the classics of Chinese culture, the wisdom of Chinese culture, and the spirit of traditional culture, but the actual content still includes life perception, parent-child education, and fashion emotions. The "spirit chicken soup" is the main, not the true spread of traditional cultural content.

Third, the main body of communication is still based on official institutions, including schools, academic research institutions, and so on. The official dissemination of natural faces is serious, professional in content, and does not attract public interest in reading. Autobiographical dissemination of individuals is often based on interests and hobbies, and content updates are not timely.

The fourth is the single form of communication, which focuses on active communication and propaganda, and the readers or users experience and interaction are very inadequate. In addition, the combination of current real-life issues is not 
close enough and the topic is not enough. As a result, it is not attractive enough to produce user stickiness.

The reasons for the above status of the dissemination mainly include the following two aspects:

The first is the change in reading groups and reading habits brought about by new media. The main users of new media are mainly young people aged 20 to 40 years. Apart from work and life, they are more inclined to and are accustomed to fragmented and entertaining reading. In such a reading environment, popular culture and popular culture characterized by "pretentiousness, inclusiveness, light mobility, easy to communicate and understand, and deep-rooted appeal" [1] are more easily accepted, followed, and disseminated.

Second, traditional culture is not flexible and popular. Due to the traditional way of traditional cultural communication, it pays great attention to the reference of classics or historical materials. Classical Chinese language skills are required for classical Chinese reading. Therefore, in most people's ideas, traditional culture is often regarded as elite culture. To a greater extent than in real life. This concept has caused both the official and the individual, the new media and the traditional media, and the traditional cultural content is often presented as "high, large, and up-to-earth." It is very alienated from people's real-life concerns and alleviates anxiety in the real world. The functions of providing spiritual entertainment and other aspects are very inadequate and can not arouse users' resonance. Therefore, the spread of new media in traditional culture has little breakthrough in the number of users and the scope of communications.

Based on the above two points, in the current situation of knowledge explosion, increasing information volume, and pressure of life and work, the focus and demand for traditional culture is more of a learning, and the reading of popular culture or popular culture content. Demand is essentially entertainment demand. Entertainment as a person's natural needs, making popular culture or on-line culture naturally take advantage of psychological and cultural needs.

\section{THE ADVANTAGES AND DISADVANTAGES OF NEW MEDIA IN THE DISSEMINATION OF EXCELLENT TRADITIONAL CHINESE CULTURE}

\section{A. Advantages}

\section{1) Fast propagation and wide coverage}

Compared to the traditional paper media and television, the new media carry the "high-speed train" of the Internet. Through the circle of friends and fans, it has completely broken the limitations of time and space, national and geographical restrictions, and can quickly deliver the content of communication. And cover a wide range. For Chinese fine traditional culture, to enhance its influence, the first step is to cover as many people as possible.
2) Strong interactivity, communication between the subject and the audience can be transformed into multiple roles

The new media platform has a powerful interactive function. The audience and media, audience and audience can use the media platform to carry out timely and smooth information interaction. In the interaction, the boundary between the communication subject and the audience is increasingly blurred and transformed. A person can become a producer, processor, distributor, and receiver of cultural information at the same time. Moreover, due to this interaction, new media can accurately analyze data for users and perform accurate content delivery and push based on the user's habits and preferences. In addition, interaction also further enhances the user stickiness of new media and provides a better mass base for the production and dissemination of cultural content.

3) The rapid development of communication technology and the rich and varied transmission carriers

With the rapid development of Internet technologies and digital technologies, new media has become more and more abundant in the form of content distribution. The performance of content can be any combination of text, pictures, video, audio, etc. The carrier can be switched between WeChat, Weibo, and APP. All these provide a good technical foundation and rich carrier form for the effective dissemination of Chinese excellent traditional culture.

\section{B. disadvantages}

\section{1) The spread of culture}

In the process of cultural communication, the new media has caused the traditional culture to be vulgarized in the production and processing of cultural content based on the consideration of commercial interests and communication effects. The names of those who advocated the culture of Chinese culture mentioned above are mainly the emotional expression and the micro-letter of the soul chicken soup. This is a concrete expression of this kitsch.

\section{2) Fragmentation of cultural systems}

Fragmented reading brings fragmentation of the dissemination of new media content. For the traditional culture, the production and dissemination of fragmented content is a great impact on the systematic nature of traditional culture, and it is easy to make the traditional culture out of context and break away from the traditional spirit.

\section{3) Longevity of dissemination is not strong}

In the era of new media, the dissemination of cultural information and content is often very time-sensitive. The continuous and massive release of information also brings about high-frequency replacement of information and hot spots. In such an environment, the dissemination of cultural information lacks persistence. This poses greater challenges to the persistence of traditional cultural transmission. 


\section{COMMUNiCATION AND INNOVATION OF CHINESE} EXCELlENT TRADITIONAL CULTURE IN THE NEW MEDia ERA

$\mathrm{Xi}$ Jin-ping pointed out that we must adapt the most basic cultural genes of the Chinese nation to contemporary culture, coordinate with modern society, and spread it in a way that people love to see and participate in. This will span time and space, surpass the country, and be rich in eternal charm. The cultural spirit of contemporary values has been promoted, and the achievements of contemporary Chinese cultural innovations that have inherited the traditional excellent culture and carried forward the spirit of the times, based on the country and the world, have been disseminated. In the era of new media, the dissemination of excellent Chinese traditional culture faces unprecedented opportunities and challenges. How to make proper use of advantages and transform disadvantages is the key to the integration of traditional culture with the requirements of the times for innovation.

\section{A. Prerequisites for the Innovation of the Spread of Chinese Excellent Traditional Culture}

1) Adhere to the guidance of socialist core values

Values are the soul and fundamental of cultural soft power, and they are the deepest elements that determine the nature and direction of culture. The vitality, cohesion, and appeal of core values are the decisive factors of the country's cultural soft power. Therefore, the orientation of values also determines the direction of cultural communication and is also the inner core of cultural communication. As the spiritual lifeblood of the Chinese nation, the excellent traditional Chinese culture is an important source of conserving socialist core values, and it is also a firm foundation for us to stand firm in the world cultural agitation. At the same time, the core value of socialism is the inheritance, development, and sublimation of traditional Chinese cultural values. To promote and disseminate the fine traditional Chinese culture, it must be carried out on the premise of adhering to the orientation of socialist core values.

\section{2) Respect Chinese traditional cultural characteristics}

Any kind of culture has its own inherent attributes and follows a certain internal development path. This is the essence of cultural preservation. In the dissemination of the new media of traditional culture, only by respecting the characteristics of the fine traditional Chinese culture can we lose our orientation and lose self in the process of catering to the tastes and needs of the general public, and we can always maintain the essential characteristics and basic connotations of traditional Chinese culture.

\section{B. Key points for the innovation and dissemination of excellent traditional Chinese culture}

1) Take the modern expression as the breakthrough point

In the era of new media, where everyone can be the main body of communication, the process of cultural communication is actually the process of communicating with others. Therefore, the way of expression is crucial. How to integrate the traditional cultural language system with the current language system and use modern language, popular and interesting expression of the essence and attitude of traditional culture is the primary breakthrough point of traditional cultural communication. The successful case of traditional media in traditional culture is the 100 -odd pulpit and poetry conference. The former uses the speaker's humorous and humorous interpretations to push the traditional classic reading into a climax, while the latter uses the form of the poetry contest to convey the long-lasting charm of classical poetry through the excellent performance of the participating players and triggers a poetry recitation. The tide. In the new media, the new media marketing conducted by the Palace Museum is through the

"selling and smashing" style of writing. With interesting and unexpected contents, it has broken the seriousness, inflexibility, and daily appearance of the Imperial Palace. [2] It has passed through the subtle changes. The cultural connotation of the cultural relics of the Forbidden City is also rich in the image of the Forbidden City. It has become fashionable and close to the people and has a deep connotation. Of course, this is done on the basis of respecting the basic connotation and spirit of the cultural relics and culture, otherwise it will lose its due spiritual tone.

2) Strengthen thematic planning, conduct topic building, and find the spiritual fit point of the era.

In the era of new media, due to changes in reading groups and reading habits, traditional culture alone cannot effectively attract users. The dissemination of new media in traditional culture must incorporate realistic social issues, combine people's feelings, combine topical planning with the topic of the times, draw on the construction of hot topics on the Internet, conduct topic construction and dissemination, and continue to convey traditional culture and value in featured topics. Orientation, and then expand the attention and influence of traditional culture. Fragmented reading, strong timeliness, and poor durability are ideal for topical marketing. However, due to the timeliness and persistence of topic creation and dissemination, if it is to achieve sustained results, it must be a planned, step-by-step continuous topic development, that is, the scientific integration and combing of related content of traditional culture, according to the topic Or the theme, find out its inner fit with the spirit of the times.

3) Strengthen the interaction and exchange, and focus on the transformation of the audience from the main body of communication.

In the era of new media, users are the main body of communication. To strengthen interactive communication is to transform the passive "audiences" into "users" or "fans" with active communication characteristics. This is one of the effective means to expand the dissemination of subjects. Especially in the process of creating and disseminating topics, timely and good interactive communication can not only control and guide the development of topics, but also spread the process of correct values. It is also an excellent second "bubbling" process. In WeChat, among the official public officials in the political and political sectors with a high degree of concern, the role of good interactive communication is often prominent. Because such public numbers represent official positions, the text is often relatively serious and legit. However, in the comments and comments, public comment editors' witty remarks and humorous responses and comments often serve as a weapon to increase user favorability and attention. . Therefore, making full use of the strong interactive features of 
new media is an important focal point for traditional culture to expand its dissemination capabilities.

\section{4) Multi-channel force, multi-format display}

One of the important foundations for the powerful dissemination of new media is the richness and diversity of the carrier of communication brought about by technological development. Making full use of related carriers and technologies such as pictures, video, audio, and VR technologies is an important way to make traditional culture lively, popular, and diverse. The once sensational "China on the tip of the tongue", although it is a traditional media, has quickly changed people's perception of Chinese traditional cuisine because of the availability of video shooting and commentary. The spirit of Chinese traditional culture is also a must for the audience. The sensory experience and the hungry appetite for food have gradually taken root. If only text, audio, or pictures, the actual results will be greatly reduced. Therefore, according to the content of traditional culture, choosing suitable, reasonable, and rich display methods and approaches will greatly increase the appeal of traditional culture, and gradually change the cultural and ecological pattern in the silently sifting.

\section{CONCLUSION}

In the era of new media, the dissemination of excellent traditional Chinese culture requires full use of the advantages of new media. Under the premise of adhering to the orientation of the core values of socialism and respecting the inherent characteristics of traditional culture, the expression of innovation is the breakthrough point, and it is constructed through theme planning. The meeting point of the spirit of the times and the value of the times has increased the transformation of communication receptors to the main body, carried out multiple forms of transformation and display, and gradually established the pattern of the spread of traditional culture and new media.

\section{REFERENCES}

[1] Rong Rong. Research on the characteristics of popular culture and its generative mechanism [J]. Journal of Tianjin Normal University: Social Science Edition, 2009, (3):57-61.

[2] Wang Wei-na. New Media Reshaping Old Tradition-Brand Innovation of Beijing Palace Museum [J]. Art Communication Research, 2016. (4): 89-96. 